Check for updates

Cite this: RSC Adv., 2020, 10, 25509

Received 9th March 2020

Accepted 22nd June 2020

DOI: $10.1039 /$ dOra05279e

rsc.li/rsc-advances

\section{The mechanochemical Scholl reaction as a versatile synthesis tool for the solvent-free generation of microporous polymers $\uparrow$}

\author{
Annika Krusenbaum, Sven Grätz, (D) Sarah Bimmermann, Stefanie Hutsch \\ and Lars Borchardt (D)*
}

\begin{abstract}
Herein we report the mechanochemical Scholl polymerization of 1,3,5-triphenylbenzene in a high speed ball mill. The reaction is conducted solvent-free, solely using solid $\mathrm{FeCl}_{3}$. The resulting porous polymer was obtained in $>99 \%$ yield after very short reaction times of only 5 minutes and exhibits a high specific surface area of $658 \mathrm{~m}^{2} \mathrm{~g}^{-1}$, which could be further enhanced up to $990 \mathrm{~m}^{2} \mathrm{~g}^{-1}$ by liquid assisted grinding. Within this study we illuminate the origin of porosity by investigating the impact of various milling parameters and milling materials, temperature and pressure, and different liquids for LAG as well as post polymer milling. Finally we expand the procedure to different monomers and mills, to present the mechanochemical Scholl reaction as a versatile synthesis tool for porous polymers.
\end{abstract}

\section{Introduction}

Microporous materials are ubiquitously applied in various fields of industrial relevance such as in heterogeneous catalysis, ${ }^{\mathbf{1 , 2}}$ gas $^{\mathbf{3}}$ and ionic charge storage, ${ }^{\mathbf{4 , 5}}$ or membrane separation. ${ }^{6}$ As these materials are defined as solids containing interconnected pores with small diameters of less than $2 \mathrm{~nm}$, they usually possess high specific surface areas of up to $3000 \mathrm{~m}^{2}$ $\mathrm{g}^{-1} \cdot{ }^{7}$ While inorganic microporous materials such as porous metal oxides ${ }^{8,9}$ or zeolites ${ }^{10}$ have already been explored thoroughly within the last decades, purely organic frameworks gain increasing research interest nowadays. Within this material group, particularly porous organic polymers (POPs) are of high significance, as they feature certain characteristics like high surface areas with adjustable pore sizes and enhanced physiochemical stability. ${ }^{\mathbf{1 1 , 1 2}}$ They can be subdivided into crystalline covalent organic frameworks (COFs) ${ }^{12,13}$ and amorphous porous aromatic frameworks (PAFs), ${ }^{14-16}$ hyper-crosslinked polymers (HCPs), ${ }^{17,18}$ polymers of intrinsic microporosity (PIMs) ${ }^{1,19}$ and conjugated microporous polymers (CMPs). ${ }^{\mathbf{6}, 20}$

The synthesis of POPs is mainly achieved by solvent-based approaches, like for example by Friedel-Crafts alkylations, ${ }^{21}$ metal-catalysed reactions ${ }^{22,23}$ e.g. Suzuki or Sonogashira-Hagihara coupling reactions, Schiff base reactions ${ }^{24,25}$ and cyclotrimerization reactions. ${ }^{26,27}$ In addition to these reactions, one important tool for the coupling of aryl systems is the Lewis acidmediated Scholl reaction. ${ }^{28-31}$ Although this reaction is known

Anorganische Chemie I, Ruhr-Universität Bochum, Universitätsstraße 150, 44801 Bochum, Germany. E-mail: lars.borchardt@ruhr-uni-bochum.de

$\dagger$ Electronic supplementary information (ESI) available. See DOI: 10.1039/d0ra05279e for certain advantages, such as the lacking requirement for expensive catalysts, starting materials or external cross linkers, there are still certain drawbacks to bypass. ${ }^{\mathbf{1 1}}$ The wet chemical approach mainly suffers from the low solubility of especially larger precursor molecules, which has to be circumvented by the introduction of solubilizing groups and results in additional synthesis steps. Furthermore, instant precipitation of the products can lead to a low degree of polymerization.

Mechanochemical synthesis concepts, such as high energy ball milling, display a versatile tool to circumvent the aforementioned drawbacks and enhance the syntheses sustainability. ${ }^{32-34}$ The transfer of mechanical energy from the colliding balls to the involved particles in a high speed ball mill results in a chemical reaction. ${ }^{35-37}$ Therefore, no solvents are utilized, which makes it possible to bypass the solubility issues of larger monomers and minimize the generated waste. ${ }^{38-40}$ Recently, we have proven feasibility and sustainability of this approach applying it to the synthesis of nanographenes, and highly porous thiophene polymers. ${ }^{\mathbf{4 1 , 4 2}}$ Furthermore, by now several mechanochemical processes for the solvent free generation of porous polymers are known. Examples are the mechanochemical synthesis of polymers of intrinsic microporosity (PIMs), ${ }^{\mathbf{4 3}}$ which are especially useful as membranes in gas separation, of COFs, ${ }^{\mathbf{4 4}}$ known for their diverse applicability in photoelectric devices, in catalysis or for gas storage, and of covalent triazine frameworks (CTFs), ${ }^{45}$ capable for selective $\mathrm{CO}_{2}$ capture, or to serve as electrode material in lithium-sulfur batteries or super capacitors. ${ }^{46}$

Within this study, we showcase how microporous polymers can be synthesized via a mechanochemical Scholl reaction in the solvent-free environment of a ball mill. During the reaction, the starting material 1,3,5-triphenylbenzene was intensely 
milled with the solid Lewis acid $\mathrm{FeCl}_{3}$. The systematic variation of the milling parameters promoted the rapid synthesis of the desired porous polymer within very short reaction times of 5 minutes and in yields of up to $>99 \%$. Additionally, it was possible to further enhance the generated surface area up to $1000 \mathrm{~m}^{2} \mathrm{~g}^{-1}$ by liquid-assisted grinding (LAG). To determine the impact of the added liquid and the $\mathrm{HCl}$ evolution, temperature and pressure measurements were performed in situ during the milling process. Overall, the mechanochemical synthesis approach provides enormous advantages in comparison to the wet chemical approach, as there is no need for harsh reaction conditions or hazardous solvents, although the Lewis acid could be substituted with an even more sustainable alternative in future. Nevertheless, the short reaction time and the easy scalability renders the mechanochemical Scholl coupling reaction as promising synthesis pathway for the generation of porous organic polymers.

\section{Experimental section}

In a typical synthesis approach, $0.540 \mathrm{~g}$ (1.77 mmol, 1 eq.) 1,3,5triphenylbenzene and $3.460 \mathrm{~g}$ (21.33 mmol, 12 eq.) $\mathrm{FeCl}_{3}$ were brought to reaction in a $50 \mathrm{ml} \mathrm{ZrO}_{2}$ grinding jar, implemented in a Retsch MM500 mixer mill. As standard reaction parameters, a milling time of $5 \mathrm{~min}$ with a frequency of $30 \mathrm{~Hz}$ and $22 \varnothing$ $=10 \mathrm{~mm} \mathrm{ZrO}_{2}$ milling balls (each ball with an average weight of $3.2 \mathrm{~g}$ ) was chosen. Subsequent to the completed reaction, the resulting solid was washed out of the jar with water. This mixture was filtered and washed with acetone to yield a black powder, which was dried at $80{ }^{\circ} \mathrm{C}$ overnight.

In another approach, the polymer synthesis was transferred to a Fritsch Pulverisette 7 premium line planetary ball mill to measure the pressure and temperature during the reaction inside the reaction vessel with a GTM (gas pressure and temperature measurement) system. For the synthesis, $22 \varnothing=$ $10 \mathrm{~mm} \mathrm{ZrO}_{2}$ milling balls (average weight of $3.2 \mathrm{~g}$ per ball) were implemented in a $45 \mathrm{ml} \mathrm{ZrO}_{2}$ milling beaker. To achieve a comparability between the products obtained by the two different milling types, the reaction was performed at $800 \mathrm{rpm}$ within 5 minutes, while the amount of implemented chemicals was kept constant. In addition to this, the workup was conducted in the same fashion as aforementioned.

The porosity of the synthesized porous polymer was investigated by nitrogen physisorption measurements, performed on a Quantachrome Quadrasorb instrument at $77 \mathrm{~K}$. Prior to the measurement, all samples were activated at $353 \mathrm{~K}$ for $24 \mathrm{~h}$ under vacuum. For physisorption measurements, high purity gases were used $\left(\mathrm{N}_{2}: 99.999 \%\right)$. The specific surface areas $\left(\mathrm{SSA}_{\mathrm{BET}}\right)$ were determined using the BET (Brunauer, Emmett, Teller) equation with the help of a micropore BET assistant. Additionally, total pore volumes were estimated using the adsorption branch at $p / p_{0}=0.95$ and pore size distributions were calculated by the DFT (Density Functional Theory) method for slit, cylindrical and sphere pores. In addition to this, the polymer was characterized by solid state ${ }^{13} \mathrm{C}$ CP-MAS NMR experiments, which were performed on the Bruker DSX 400 spectrometer, equipped with a VTN double resonance max. $35 \mathrm{kHz} 2.5 \mathrm{~mm}$ MAS ${ }^{1} \mathrm{H}$ XBB probe head. The samples were rotated at $10 \mathrm{kHz}$ and measured with ramped ${ }^{1} \mathrm{H}-{ }^{13} \mathrm{C}$ cross polarization. As reference for the peak assignments, an adamantane probe was utilized. Infrared spectroscopy (IR) was carried out on a SHIMADZU IRSpirit Fourier transform infrared spectrometer equipped with a single reflection ATR unit. Thermogravimetric analysis (TGA) and simultaneous differential thermal analysis (DTA) were performed on a Seiko TG 6200/SII. For each measurement, $10 \mathrm{mg}$ of the respective compound were filled into an aluminium crucible. Afterwards, the crucible was immersed in a constant nitrogen flow (300 $\left.\mathrm{ml} \mathrm{min}^{-1} ; 99.999 \%\right)$ inside

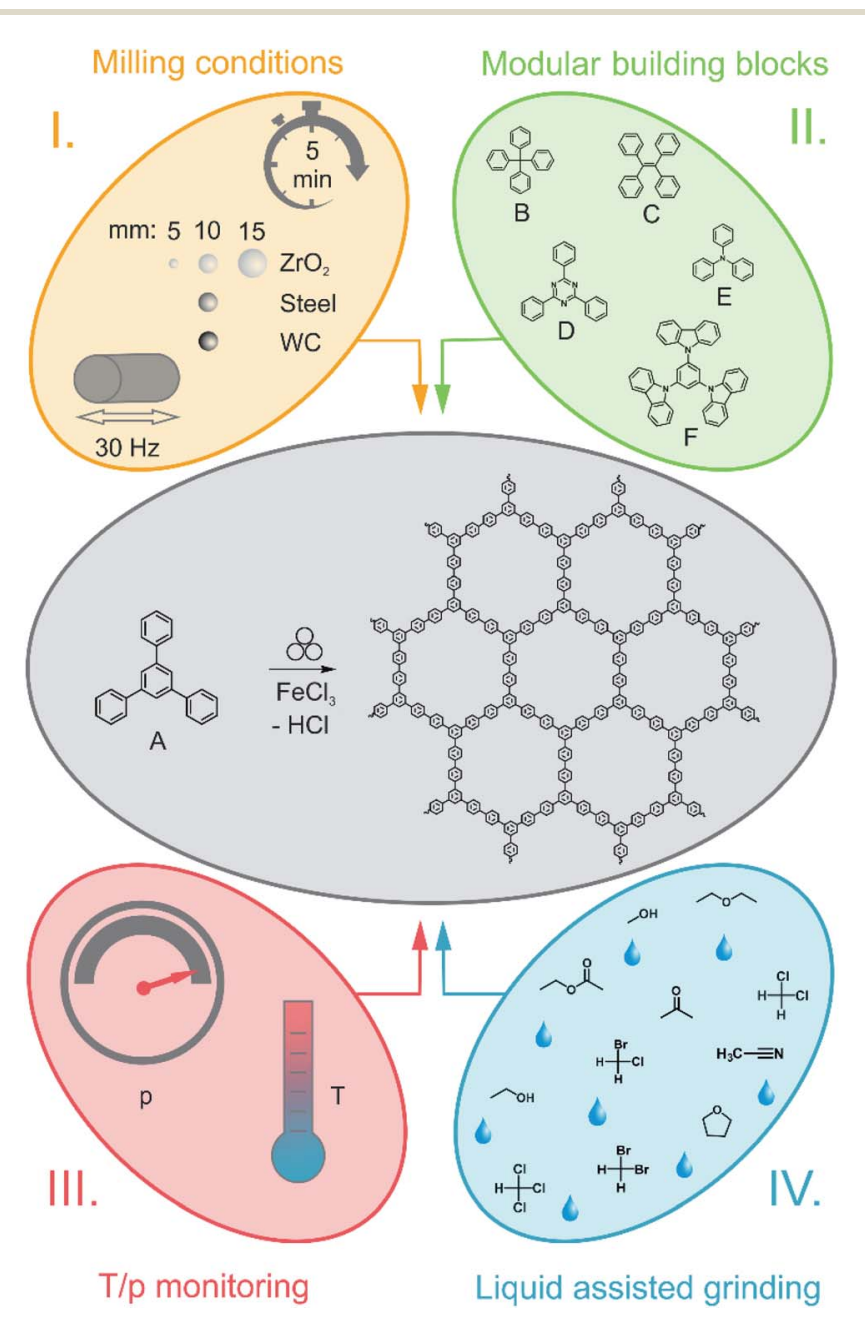

Fig. 1 Schematic overview over the parameter variation for the Scholl reaction. The middle part shows the standard reaction in a MM500 mixer mill at $30 \mathrm{~Hz}$ for $5 \mathrm{~min}$. For the reaction, the monomer $\mathrm{A}(1,3,5-$ triphenylbenzene) is used. I. Systematic variation of milling parameters, as for instance the time, frequency and size and density of the milling material. II. Variation of monomers used for the Scholl reaction. For the reaction, the monomers $B$ (tetraphenylmethane), C (tetraphenylethylene), D (2,4,6-triphenylbenzene-1,3,5-triazine), E (triphenylamine) and $F(1,3,5$-tris $(N$-carbazolyl)benzene) were used. III. The variation of the temperature during the reaction. Temperature and pressure were recorded during the reaction using a gas pressure and temperature measurement (GTM) system. IV. The Scholl reaction with liquid assisted grinding (LAG). 
the machine and measured with a heating rate of $5{ }^{\circ} \mathrm{C} \mathrm{min}^{-1}$ in a temperature regime between $30^{\circ} \mathrm{C}$ and $550{ }^{\circ} \mathrm{C}$. Elemental Analysis (EA) was carried out by the Elementar vario MIRCROcube instrument from Elementar. The composition of the sample was determined with respect to the carbon, nitrogen, hydrogen and sulphur content. Rutherford backscattering spectrometry (RBS) was performed at RUBION (Central Unit for Ion Beams and Radionuclides at Ruhr-University Bochum). Therefore, a $2.0 \mathrm{MeV}{ }^{4} \mathrm{He}^{+}$ion beam (intensity 20-40 nA) was directed towards the sample at $7^{\circ}$ and the backscattered particles were detected at $160^{\circ}$ (Si detector, resolution $=16 \mathrm{keV}$ ). Powder X-ray diffraction (PXRD) was investigated with a Bruker D2 PHASER spectrometer with $\mathrm{CuK} \alpha(1.54184 \AA)$ radiation. UV/VIS absorption spectra were recorded with the Jasco V-670 spectrometer between 300$2500 \mathrm{~nm}$. SEM images were recorded, using a high-resolution scanning electron microscope (JEOL $7500 \mathrm{~F}$ ) at $5 \mathrm{kV}$ and with a Hitachi SU8020 SEM equipped with a secondary electron (SE) detector at $2 \mathrm{kV}$.

\section{Results and discussion}

\section{Mechanochemical Scholl reaction of 1,3,5-triphenylbenzene}

The mechanochemical Scholl polymerisation of 1,3,5-triphenylbenzene was carried out exemplarily under the described conditions and serves as reference system. The resulting product is a porous polymer and will be declared as PP1 (porous polymer 1) from now on. In order to ensure the reproducibility of this reaction, we have repeated the standard reaction five times. During this investigation, the specific surface area (SSA ${ }_{\mathrm{BET}}$ ) was varying between $640-658 \mathrm{~m}^{2} \mathrm{~g}^{-1}$, which corresponds to a discrepancy of $2.7 \%$, while the yield was alternating between 99 and $>99 \%$.

During the polymerization, a colour change from pale yellow (monomer) to dark brown (polymer) occurred (Fig. 2(1)). The CP-MAS NMR spectrum of the polymer (Fig. 2(2)) exhibits two broad resonance peaks at $\delta=140 \mathrm{ppm}$ and $\delta=126 \mathrm{ppm}$, which can be assigned to the coupled aromatic carbons a, $\mathrm{d}$ and e and to the non-coupled aromatic carbons b, c and $\mathrm{f}$, respectively, and were already observed for the 1,3,5-triphenylbenzene
(1)

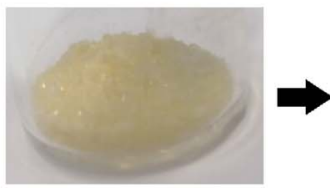

1,3,5-Triphenylbenzene

(3)

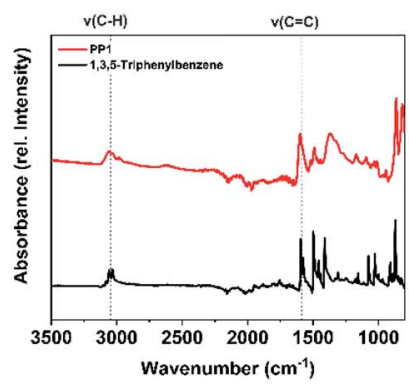

(6)

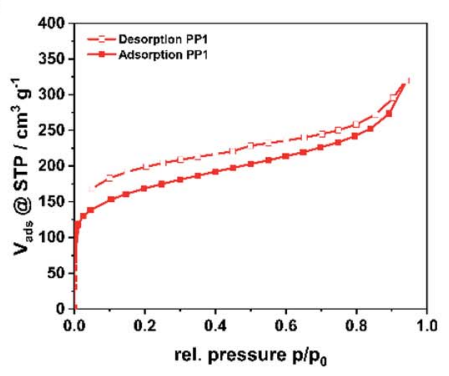

(2)

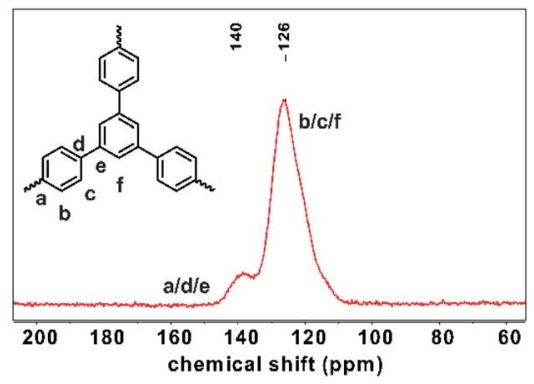

(4)

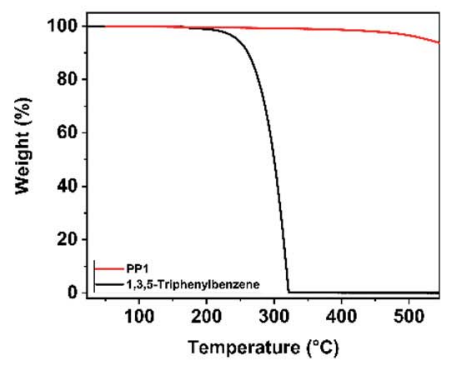

(7)

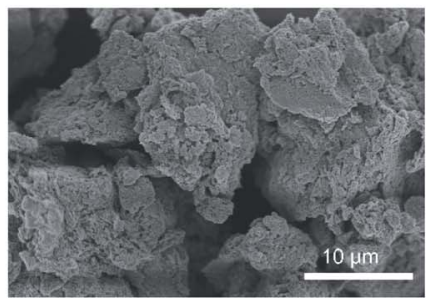

(5)
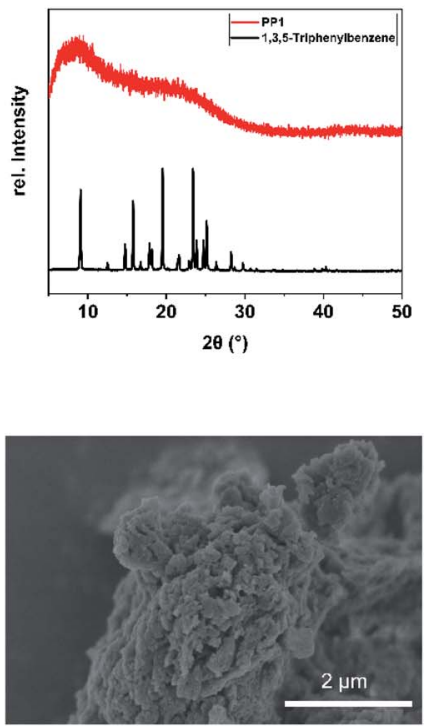

Fig. 2 Analysis of the porous polymer PP1. (1) Image of the monomer in comparison to the polymer. (2) ${ }^{13} \mathrm{C}$ cross-polarization magic-angle spinning (CP-MAS) NMR of the porous polymer PP1. The peaks are assigned to the spectrum. (3) FT-IR spectra of PP1 (red) and 1,3,5-triphenylbenzene (black). The absorption band of the $\mathrm{C}-\mathrm{H}$ and $\mathrm{C}=\mathrm{C}$ stretching vibration is highlighted. (4) Thermogravimetric analysis (TGA) of PP1 (red) in comparison to the monomer 1,3,5-triphenylbenzene (black). (5) Powder X-ray diffractogram (PXRD) of PP1 (red) compared with the monomer 1,3,5-triphenylbenzene (black), showing the amorphous behaviour of the polymer. (6) Nitrogen physisorption isotherm of the porous polymer PP1 exhibiting a polymer-like swelling behaviour. (7) SEM image of the sample PP1 with a magnitude of 5000 (left) and 30000 (right). 
polymer synthesized from hot $\mathrm{CHCl}_{3}$ under inert gas atmosphere and $\mathrm{AlCl}_{3}$ as Lewis acid. ${ }^{30}$ FT-IR investigations (Fig. 2(3)) show the intact $\mathrm{C}-\mathrm{H}$ (benzene) and $\mathrm{C}=\mathrm{C}$ (benzene) vibrations in the monomer 1,3,5-triphenylbenzene and in the polymer PP1, as in the region of $3056 \mathrm{~cm}^{-1}$ a weak absorption band is visible, which is attributed to the $\mathrm{C}-\mathrm{H}$ (benzene) stretching vibration, while at $1592 \mathrm{~cm}^{-1}$ the $\mathrm{C}=\mathrm{C}$ (benzene) stretching vibration can be seen, which is assignable to the aromatic ring skeleton vibration. The observations are consistent with the FT-IR spectrum of 1,3,5-triphenylbenzene polymer synthesized by the solution based approach. ${ }^{30}$ The Scholl polymer shows high thermal stability of up to $500{ }^{\circ} \mathrm{C}$ (Fig. 2(4)). In comparison to the crystalline monomer 1,3,5-triphenylbenzene, the polymer PP1 exhibits an amorphous structure, which can be attributed to multiple stacking faults of the polymeric networks in the bulk material (Fig. 2(5)), to a distortion of the structure through the biphenyl axis, or to interpenetration. Moreover, possible crosslinking and side reactions of the forming polymer skeleton, particularly at longer reaction times, lead to structural irregularities, hence to a deviation from the schematical structure (Fig. 1) and thus explains its amorphous structure. To verify the successful intermolecular coupling reactions, UV/VIS spectroscopy was performed to neglect the occurrence of competing intramolecular reactions, which would result in the formation of fluorene units. These would feature two broad absorption bands in the UV/VIS at $303 \mathrm{~nm}$ and at $314 \mathrm{~nm}$, which are not present in the spectrum of PP1 (Fig. S3 $\dagger$ ). ${ }^{47}$ The nitrogen physisorption (Fig. 2(6)) isotherms can be assigned to IUPAC type I, which is characteristic for microporous materials. With use of the Brunauer-Emmett-Teller-equation it was possible to determine the specific surface area of the PP1 to $658 \mathrm{~m}^{2} \mathrm{~g}^{-1}$. Additionally, the total pore volume was calculated from the adsorption branch at $p / p_{0}=0.95$ to $0.53 \mathrm{~cm}^{3} \mathrm{~g}^{-1}$ and the pore width was found to be $0.64 \mathrm{~nm}$ with a fitting error of $0.3 \%$. In comparison to the solution based approach (exhibiting a surface area of $1254 \mathrm{~m}^{2} \mathrm{~g}^{-1}$ ), the specific surface area is smaller, nevertheless it was possible to further enhance the $\mathrm{SSA}_{\mathrm{BET}}$ by liquid assisted grinding, yielding a comparable value (see: Impact of liquid-assisted grinding). SEM images of the porous polymer reveal the agglomeration of smaller, unevenly shaped particles to bigger flakes (Fig. 2(7)). To determine the elemental composition of the synthesised polymer, elemental analysis (EA) was performed. The obtained values are in a range with the expected, which indicates a high purity of the polymer (Table S3†). To investigate the degree of contamination, Rutherford backscattering spectrometry was performed. On the basis of 61 at $\% \mathrm{C}$ and 38 at $\% \mathrm{H}$, the total contamination of $\mathrm{Cl}(0.94$ at $\%)$ and of $\mathrm{Fe}(0.04$ at\%) was found to be below 1 at\%. Due to this, the workup with water and acetone was fond to be promising to remove the traces of unreacted monomer as well as of the Lewis acid $\mathrm{FeCl}_{3}$.

\section{Influence of the milling parameters frequency, time and milling material}

During the process development, the systematic variation of milling parameters of the reference system PP1 was a key technique to obtain the highest possible yield with the highest possible $\mathrm{SSA}_{\mathrm{BET}}$ within the shortest possible time. Therefore, the milling time was varied in a first attempt, while the frequency was kept constant at $30 \mathrm{~Hz}$. The results are presented in Table 1 as PP1-PP7.

The Scholl polymerization of 1,3,5-triphenylbenzene is incomplete after very short reaction times of 1 or 2 minutes (PP2 and PP3). Within this time, the formation of the polymer was only achieved with $19 \%$ and $51 \%$ yield and low specific surface areas of $87 \mathrm{~m}^{2} \mathrm{~g}^{-1}$ and $61 \mathrm{~m}^{2} \mathrm{~g}^{-1}$, respectively. During the variation of milling time, the reaction was most promising for short reaction times of 5 minutes, as the polymer PP1 was already obtainable in $>99 \%$ yield. Furthermore, this short reaction exhibits the highest $\mathrm{SSA}_{\mathrm{BET}}$ of $658 \mathrm{~m}^{2} \mathrm{~g}^{-1}$. Longer milling lead to a partial degradation of the porosity, probably due to the high energy input.

The mechanochemical Scholl polymerization is very sensitive towards the energy input, as the high energy transferred from the milling balls to the particles inside the grinding jar at

Table 1 Yields, specific surface areas $\left(\mathrm{SSA}_{\mathrm{BET}}\right)$ and pore volume for the polymers obtained at different milling times and frequencies. The total pore volume $V_{\text {total }}$ was calculated from the $\mathrm{N}_{2}$ isotherm at $p / p_{0}=0.95,77 \mathrm{~K}$

\begin{tabular}{|c|c|c|c|c|c|c|}
\hline Polymer & Material & Time (min) & Frequency $(\mathrm{Hz})$ & Yield (\%) & $\operatorname{SSA}_{\text {BET }}\left(\mathrm{m}^{2} \mathrm{~g}^{-1}\right)$ & $V_{\text {total }}\left(\mathrm{cm}^{3} \mathrm{~g}^{-1}\right)$ \\
\hline PP1 & $\mathrm{ZrO}_{2}(10 \mathrm{~mm})$ & 5 & 30 & $>99$ & 658 & 0.53 \\
\hline PP2 & $\mathrm{ZrO}_{2}(10 \mathrm{~mm})$ & 1 & 30 & 19 & 87 & 0.17 \\
\hline PP3 & $\mathrm{ZrO}_{2}(10 \mathrm{~mm})$ & 2 & 30 & 51 & 61 & 0.09 \\
\hline PP4 & $\mathrm{ZrO}_{2}(10 \mathrm{~mm})$ & 10 & 30 & $>99$ & 348 & 0.28 \\
\hline PP5 & $\mathrm{ZrO}_{2}(10 \mathrm{~mm})$ & 15 & 30 & $>99$ & 505 & 0.40 \\
\hline PP6 & $\mathrm{ZrO}_{2}(10 \mathrm{~mm})$ & 30 & 30 & $>99$ & 568 & 0.61 \\
\hline PP7 & $\mathrm{ZrO}_{2}(10 \mathrm{~mm})$ & 60 & 30 & $>99$ & 421 & 0.33 \\
\hline PP8 & $\mathrm{ZrO}_{2}(10 \mathrm{~mm})$ & 5 & 10 & 26 & 17 & 0.06 \\
\hline PP9 & $\mathrm{ZrO}_{2}(10 \mathrm{~mm})$ & 5 & 20 & 44 & 87 & 0.13 \\
\hline PP10 & $\mathrm{ZrO}_{2}(10 \mathrm{~mm})$ & 5 & 25 & 75 & 111 & 0.18 \\
\hline PP11 & $\mathrm{ZrO}_{2}(10 \mathrm{~mm})$ & 5 & 35 & $>99$ & 273 & 0.24 \\
\hline PP12 & $\mathrm{WC}(10 \mathrm{~mm})$ & 5 & 30 & $>99$ & 581 & 0.43 \\
\hline PP13 & Steel $(10 \mathrm{~mm})$ & 5 & 30 & $>99$ & 499 & 0.36 \\
\hline PP14 & $\mathrm{ZrO}_{2}(5 \mathrm{~mm})$ & 5 & 30 & 83 & 285 & 0.12 \\
\hline PP15 & $\mathrm{ZrO}_{2}(15 \mathrm{~mm})$ & 5 & 30 & $>99$ & 457 & 0.36 \\
\hline
\end{tabular}


$35 \mathrm{~Hz}$ (i.e. only an increase of $5 \mathrm{~Hz}$ compared to PP1) results in a severe degradation of the porosity (sample PP11 in Table 1). However, also lower frequencies are not favourable, as the lower energy impact leads to an incomplete reaction with yields far below 100\% (PP8-PP10). Due to this, the optimal frequency for the mechanochemical Scholl reaction in a MM500 mixer mill was determined to be $30 \mathrm{~Hz}$ (PP1).

The density of the milling material is an important factor for the generation of porous polymers. If the density is too low (e.g. $\mathrm{Si}_{3} \mathrm{~N}_{4}$ ), the energy impact might not be sufficient, which hinders a fast reaction necessary for the formation of the pores. Very high density (e.g. WC) milling material is not only providing a sufficient amount of energy to the system, but also leads to a heating of the milling jar, which increases the pressure inside the vessel. Nevertheless, the energy impact might also be too high and destroy the readily formed polymers. To proof this suggestion, $22 \varnothing=10 \mathrm{~mm}$ WC or steel balls were implemented in a steel beaker under standard conditions (sample PP12 and PP13 in Table 1). Similar to the mechanochemical Scholl reaction at a higher frequency or for longer milling times, the use of higher density materials leads to a degradation of the surface area. Therefore, $\mathrm{ZrO}_{2}$ milling balls with a medium density were found to be perfectly suitable for our approach.

In a last approach, the ball size of the $\mathrm{ZrO}_{2}$ balls was varied to $\varnothing=5 \mathrm{~mm}$ and to $\varnothing=15 \mathrm{~mm}$ (sample PP14 and PP15 in Table 1). To ensure comparability, the same total ball masses were used. This corresponds to $174 \mathrm{ZrO}_{2}$ milling balls of $\varnothing=5 \mathrm{~mm}$ and 6 $\mathrm{ZrO}_{2}$ milling balls of $\varnothing=15 \mathrm{~mm}$, respectively. Again, $\varnothing=$ $10 \mathrm{~mm}$ sized balls appear to be optimal, with respect to the porosity of the obtained polymer.

To investigate how the high energy input affects the porosity of the product, we conducted post polymer milling for 30 minutes at $30 \mathrm{~Hz}$. Therefore, the porous polymer PP1 was milled with 12 eq. of $\mathrm{FeCl}_{3}$ in one attempt and with the same amount of an inert bulk material ( $\mathrm{NaCl})$ in another approach. During the milling process with $\mathrm{FeCl}_{3}$, the surface area was degraded to 560 $\mathrm{m}^{2} \mathrm{~g}^{-1}$, which corresponds to $85 \%$ of the former polymer. For the milling with $\mathrm{NaCl}$ as bulk material, the surface area changed to $432 \mathrm{~m}^{2} \mathrm{~g}^{-1}$, which equals $66 \%$ of the starting material. This confirms that the milling itself is degrading the porosity of the formed polymer and reinforces the need of short but intense milling protocols for the synthesis of porous polymers.

\section{Monomers impact}

To explore the origin of porosity, the impact of the monomer was examined. For the investigation, six different monomers were utilized in a standard reaction procedure, while the reaction parameters were kept constant (addition of 12 eq. $\mathrm{FeCl}_{3}$, $30 \mathrm{~Hz}, 5 \mathrm{~min})$. The different monomers are summarized in Table 2 as A-F.

The monomer exchange led to a broad range of porosity of the resulting polymers from non-porous (PP18) to highly porous (PP20) materials. We believe the electron density of the respective aryl compounds to impact the Scholl reaction activity and thus the development of porosity. Electron deficient systems such as 2,4,6-triphenyl-1,3,5-triazine (monomer D),
Table 2 Yields, specific surface areas (SSA $\left.A_{B E T}\right)$ and total pore volumes $\left(V_{\text {total }}\right)$ for the Scholl polymerization of different monomers

\begin{tabular}{llcll}
\hline Monomer & Polymer & Yield $(\%)$ & SSA $_{\text {BET }}\left(\mathrm{m}^{2} \mathrm{~g}^{-1}\right)$ & $V_{\text {total }}\left(\mathrm{cm}^{3} \mathrm{~g}^{-1}\right)$ \\
\hline A & PP1 & $>99$ & 658 & 0.53 \\
B & PP16 & 12 & 225 & 0.24 \\
C & PP17 & 86 & 88 & 0.24 \\
D & PP18 & 78 & n.p. & - \\
E & PP19 & 48 & 161 & 0.56 \\
F & PP20 & $>99$ & 1408 & 0.95
\end{tabular}

polymerize in yields of $78 \%$ and remain non-porous while electron rich aryl systems such as 1,3,5-triphenylbenzene (monomer A) or 1,3,5-tris( $N$-carbazolyl)benzene (monomer F) convert to porous structures with $>99 \%$ yield. The high $\mathrm{SSA}_{\mathrm{BET}}$ of the reference system PP1 of $658 \mathrm{~m}^{2} \mathrm{~g}^{-1}$ was solely exceeded by polymer PP20, which was synthesized from 1,3,5-tris $(N$-carbazolyl)benzene (monomer F) and exhibits a $\mathrm{SSA}_{\mathrm{BET}}$ of $1408 \mathrm{~m}^{2}$ $\mathrm{g}^{-1}$ (Table 2).

For all polymers, characteristic type I isotherms were observed during nitrogen physisorption at $77 \mathrm{~K}$ (Fig. 3), which are representative for microporous materials. The isotherms of the polymers gained from monomer $\mathrm{C}$ and $\mathrm{E}$ furthermore feature a high nitrogen uptake at $p / p_{0}=0.95$, which is attributed to a high inter-particular space within the sample. All
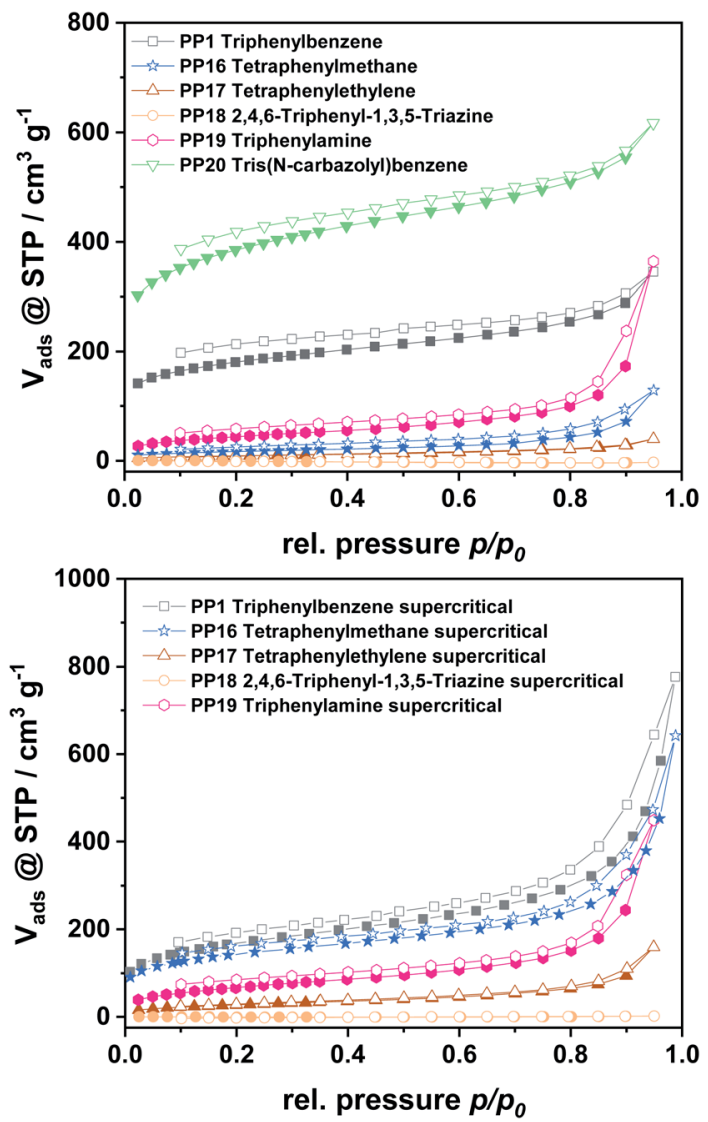

Fig. $3 \quad \mathrm{~N}_{2}$ physisorption isotherms for polymers dried at $80{ }^{\circ} \mathrm{C}$ (top) and for supercritically dried polymers (bottom). 
isotherms feature a certain non-reversibility of the adsorption and desorption branch, which is due to the swelling-behaviour of the amorphous polymers. For a deeper insight into the pore formation, the polymers obtained by the different monomers were also dried supercritically to prevent pore collapse during the drying procedure. Due to this it was possible to expand the obtained surface areas by a factor of $1.5-2$, as the original pore structure is kept intact during drying.

\section{The impact of temperature and pressure on the reaction}

The collision of milling balls with the grinding vessel walls and each other results in a step by step heating of the milling vessel.

To investigate the impact of the temperature on the mechanochemical Scholl reaction, the reference system was milled at different temperatures. Since a direct regulation of the milling temperature is hardly feasible at the moment we filled and closed the steel milling vessels at room temperature and then transferred them into an oven or refrigerator overnight to achieve a homogenous temperature inside the vessels. Since the reaction time is short, we assumed that the temperature we achieved with external heating or cooling is not changing too much during the milling process, while the milling itself leads to a stepwise increase of the vessel temperature. However, as this temperature increase due to the milling itself is occurring for every reaction, it can be neglected in the given case.

It seems that the reaction is proceeding slower at lower temperatures, leading to lower yields and smaller SSA $\mathrm{BET}_{\text {(174 }}$ $\mathrm{m}^{2} \mathrm{~g}^{-1}$, PP21). At elevated temperatures higher surface areas could be achieved $\left(657 \mathrm{~m}^{2} \mathrm{~g}^{-1}\right.$, PP26) up to an external temperature of $100{ }^{\circ} \mathrm{C}$. The temperature probably also has an indirect impact on the system, as a higher temperature leads to a further pressure increase inside the milling jar (Table 3).

To exhibit the impact of pressure on the system, we had to transfer the reaction to a Fritsch Pulverisette 7 premium line planetary ball mill (P7) operated with a gas pressure and temperature measurement (GTM) system at 800 rpm. Transferring the reaction from a vibrational to a planetary ball mill was possible as the polymers obtained from both mills are comparable among each other (for further information please see Table S2 $\uparrow$ ). For this analysis the milling time was elongated to up to one hour in order to be able to assess the whole pressure and temperature development. For PP1 the temperature is

Table 3 Yield, specific surface areas (SSA $\left.\mathrm{BET}_{\mathrm{BET}}\right)$ and total pore volume $\left(V_{\text {total }}\right)$ for the milling of the reference system at different temperatures inside steel vessels

\begin{tabular}{llcll}
\hline Polymer & Temperature $\left({ }^{\circ} \mathrm{C}\right)$ & Yield $(\%)$ & SSA $_{\text {BET }}\left(\mathrm{m}^{2} \mathrm{~g}^{-1}\right)$ & $V_{\text {total }}\left(\mathrm{g} \mathrm{cm}^{-3}\right)$ \\
\hline PP21 & -50 & 68 & 174 & 0.36 \\
PP22 & -20 & 89 & 195 & 0.19 \\
PP23 & 0 & 95 & 359 & 0.38 \\
PP13 & RT & $>99$ & 499 & 0.36 \\
PP24 & 50 & $>99$ & 595 & 0.53 \\
PP25 & 75 & $>99$ & 568 & 0.47 \\
PP26 & 100 & $>99$ & 657 & 0.44 \\
PP27 & 125 & $>99$ & 522 & 0.41
\end{tabular}

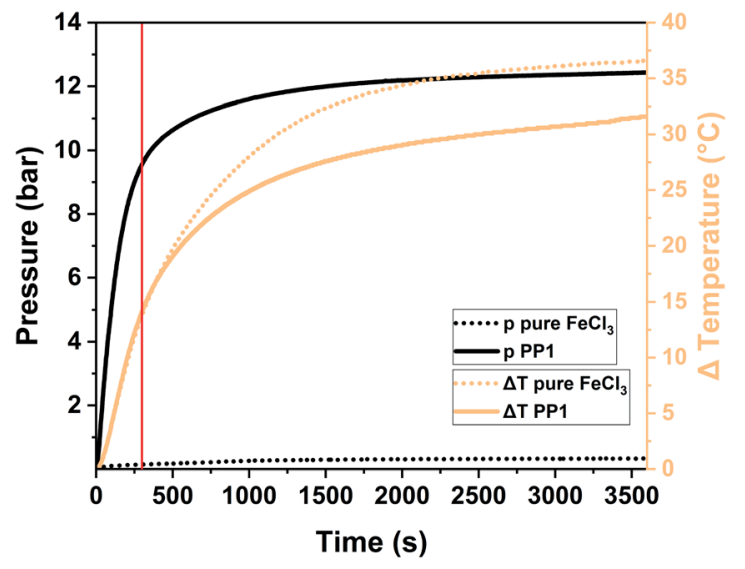

Fig. 4 Pressure and temperature profile of the Scholl reaction of the sample PP1 (solid line) in comparison to the grinding of pure $\mathrm{FeCl}_{3}$ (dotted line). The standard reaction time of 5 minutes is assigned to the spectrum as red, solid line.

increasing to $37^{\circ} \mathrm{C}\left(\Delta T=14{ }^{\circ} \mathrm{C}\right)$ after 5 minutes (marked with a red line in Fig. 4) and to $55^{\circ} \mathrm{C}\left(\Delta T=32^{\circ} \mathrm{C}\right)$ after one hour of milling. While the temperature increase for the milling of pure $\mathrm{FeCl}_{3}$ and of the sample PP1 (1 eq. 1,3,5-triphenylbenzene +12 eq. $\mathrm{FeCl}_{3}$ ) was almost identical, there are huge differences between the pressure developments of the same systems. During the milling of pure $\mathrm{FeCl}_{3}$ no reaction takes place, therefore no $\mathrm{HCl}$ evolution is occurring. The small increase in pressure to 380 mbar during the milling is attributed to the temperature increase and therefore to the expansion of air captured inside the sealed milling vessel. For the milling of the sample PP1 a steep pressure increase to $\sim 9.5$ bar was observed during the first 5 minutes of the measurements. Afterwards the curve flattens, as the reaction and therefore the evolution of $\mathrm{HCl}$ is almost completed. After one hour the overall pressure inside the vessel is settled at $\sim 12$ bar.

\section{Impact of liquid-assisted grinding}

Based on our previous results on hyper-crosslinked polymers, we knew that small quantities of liquid (liquid assisted grinding, LAG) can greatly impact the porosity of polymers. ${ }^{\mathbf{1 7}}$ Therefore, we started to add a varying amount of dichloromethane (DCM) to the grinding vessel.

The addition of small quantities of DCM enhanced the specific surface area of the polymer to more than $1050 \mathrm{~m}^{2} \mathrm{~g}^{-1}$. Larger quantities, i.e. $2 \mathrm{ml}$, of liquid led to a degradation of the porosity again. This behaviour reveals that the enhanced surface area for the addition of small quantities of DCM is not a solvation effect, however the liquid rather serves as catalyst or mediator.

The systematic variation of different liquids added to the grinding jar was conducted to examine the origin of porosity during the reaction (Table 4). In the following, the liquid assisted grinding was performed for up to 1 hour to ensure an adequate in situ temperature and pressure monitoring.

At first, we assumed that the liquid may act as solvent for the evolving $\mathrm{HCl}$ during the reaction, which would therefore be captured and thus impact the development of porosity of the 
Table 4 Yields, specific surface areas (SSA $\left.\mathrm{BET}_{\mathrm{B}}\right)$ and total pore volumes $\left(V_{\text {total }}\right)$ for liquid assisted grinding with varying amounts of different liquids at 800 rpm in the planetary ball mill P7. The top part (PP28-PP31) exhibits the results for the reaction for 5 minutes and the lower part (PP32PP42) shows the milling operated for up to 1 hour for in situ measurements of pressure and temperature

\begin{tabular}{|c|c|c|c|c|c|}
\hline Polymer & Liquid & Amount (ml) & Yield (\%) & $\operatorname{SSA}_{\mathrm{BET}}\left(\mathrm{m}^{2} \mathrm{~g}^{-1}\right)$ & $V_{\text {total }}\left(\mathrm{cm}^{3} \mathrm{~g}^{-1}\right)$ \\
\hline PP28 & DCM & 0.5 & $>99$ & 1069 & 0.72 \\
\hline PP29 & DCM & 1 & 97 & 1090 & 0.73 \\
\hline PP31 & DCM & 2 & $>99$ & 733 & 0.52 \\
\hline PP32 & DCM & 1 & $>99$ & 998 & 0.68 \\
\hline PP33 & $\mathrm{CHCl}_{3}$ & 1 & $>99$ & 845 & 0.58 \\
\hline PP36 & $\mathrm{Et}_{2} \mathrm{O}$ & 1 & 90 & 55 & 0.01 \\
\hline PP37 & EtOH & 1 & $>99$ & 173 & 0.10 \\
\hline PP38 & EtOAc & 1 & $>99$ & 318 & 0.27 \\
\hline PP39 & $\mathrm{MeCN}$ & 1 & $>99$ & 72 & 0.15 \\
\hline PP40 & $\mathrm{MeOH}$ & 1 & 76 & 22 & 0.07 \\
\hline PP41 & Acetone & 1 & $>99$ & 39 & 0.05 \\
\hline
\end{tabular}

obtained polymer. However, the solubility of $\mathrm{HCl}$ in $\mathrm{CHCl}_{3}$ and in DCM is significantly lower than in $\mathrm{Et}_{2} \mathrm{O}$, which is contrary to the obtained surface areas (PP32, PP33, PP36). In the following, we hypothesized that the boiling point of the liquid impacts pore formation as the vapour may serve as porogen. Again, we can rule out this, as the similarity of the boiling points of DCM $\left(40{ }^{\circ} \mathrm{C}\right)$ and of $\mathrm{Et}_{2} \mathrm{O}\left(35^{\circ} \mathrm{C}\right)$ leads to materials with greatly different surface areas of 998 and $55 \mathrm{~m}^{2} \mathrm{~g}^{-1}$, respectively. Since the porosity of the polymer is greatly enhanced by LAG with $\mathrm{CHCl}_{3}$ and DCM, it has to be taken into consideration that these chemicals might not only act as a solvent for $\mathrm{HCl}$, but are part of the reaction process. One suggestion would be a competing Friedel-Crafts reaction, which provides methylene cross-links and enhances the porosity of the polymer. Nevertheless, this was disproved as well, as ${ }^{13} \mathrm{C}$ CP-MAS NMR analysis showed no additional signals originating from a methylene cross-linker (see Fig. S5 $\dagger$ ). During the investigation, solely halogenated liquids resulted in an enlargement of the surface area (PP32PP35), while the addition of non-halogenated liquids led to a surface area degradation (PP36-PP42). It is presumable that the halogenated liquid and $\mathrm{FeCl}_{3}$ associate, ${ }^{48}$ which results in a highly Lewis acidic $\mathrm{FeCl}_{2}{ }^{+}$intermediate that is very reactive. ${ }^{\mathbf{4 9 5 0}}$ This might accelerate the reaction further leading to a higher cross-linking, disorder and thus higher porosity. Interestingly, during the LAG with different liquids, the addition of halogenated liquids gives higher pressures than the addition of non-halogenated liquids, which correlates well with the obtained surface areas (see Fig. S11-S23†). Therefore, we postulate that the high pressure can be attributed to an increased $\mathrm{HCl}$ evolution evoked from the highly Lewis acidic $\mathrm{FeCl}_{2}{ }^{+}$intermediate, which is increasing the specific surface area of the polymer.

\section{Conclusion}

Herein we reported the mechanochemical Scholl polymerization of 1,3,5-triphenylbenzene with solid $\mathrm{FeCl}_{3}$ in a MM500 mixer mill. Our approach is not relying on the use of any solvents, which makes the synthesis unhazardous and more sustainable than the classical Scholl polymerization, performed under the use of inert gas atmosphere and hot $\mathrm{CHCl}_{3}$. It was even possible to obtain the desired porous polymer within very short reaction times of only 5 minutes and in yields of up to $>99 \%$, which makes the former used multistep synthesis with reaction times of $48 \mathrm{~h}$ and a $24 \mathrm{~h}$ Soxhlet extraction neglectable. In addition to this, it was possible to transfer the synthesis approach to different monomers, yielding polymers with specific BET surface areas of more than $1400 \mathrm{~m}^{2} \mathrm{~g}^{-1}$. During the study, the pore formation was examined with regards to the drying procedure, the milling-parameters and -materials and the temperature and pressure inside the reaction vessel. Furthermore, the impact of liquid assisted grinding (LAG) was examined with respect to the evolution of $\mathrm{HCl}$ during the reaction. Summarizing, the mechanochemical Scholl reaction is a versatile tool for the fast, easy and sustainable synthesis of porous polymers, which are very important in several industrial applications nowadays.

\section{Conflicts of interest}

There are no conflicts to declare.

\section{Acknowledgements}

The author gratefully acknowledges the Federal Ministry of Education and Research (Bundesministerium für Bildung und Forschung, BMBF) for support of the Mechanocarb project (award number 03SF0498). Additionally, the author acknowledges Nils Boysen for TGA/DTA measurements, Lisa Feldhaus for EA measurements, Dr Detlef Rogalla for RBS measurements and Dr Carsten Schauerte for PXRD measurements. Furthermore, special thanks go to Dr Cathleen Oschatz and Christian Vogt for the recording of SEM images, to Hans-Jochen 
Hauswald for assistance at the ${ }^{13} \mathrm{C}$ CP-MAS NMR measurements and to David Tetzlaff for UV/VIS measurements.

\section{References}

1 N. B. McKeown and P. M. Budd, Chem. Soc. Rev., 2006, 35, 675.

2 Y.-B. Zhou and Z.-P. Zhan, Chem.-Asian J., 2018, 13, 9.

3 A. Li, R.-F. Lu, Y. Wang, X. Wang, K.-L. Han and W.-Q. Deng, Angew. Chem., Int. Ed., 2010, 49, 3330.

4 C. Prehal, S. Grätz, B. Krüner, M. Thommes, L. Borchardt, V. Presser and O. Paris, Carbon, 2019, 152, 416.

5 L. Wang, J. Ding, S. Sun, B. Zhang, X. Tian, J. Zhu, S. Song, B. Liu, X. Zhuang and Y. Chen, Adv. Mater. Interfaces, 2018, 5, 1701679.

6 J.-X. Jiang, F. Su, A. Trewin, C. D. Wood, N. L. Campbell, H. Niu, C. Dickinson, A. Y. Ganin, M. J. Rosseinsky, Y. Z. Khimyak, et al., Angew. Chem., Int. Ed., 2007, 46, 8574.

7 F. Schüth, K. S. W. Sing and J. Weitkamp, Handbook of Porous Solids, Wiley, 2002.

8 A. Huang, Y. He, Y. Zhou, Y. Zhou, Y. Yang, J. Zhang, L. Luo, Q. Mao, D. Hou and J. Yang, J. Mater. Sci., 2019, 54, 949.

9 W. Xiao, S. Yang, P. Zhang, P. Li, P. Wu, M. Li, N. Chen, K. Jie, C. Huang, N. Zhang, et al., Chem. Mater., 2018, 30, 2924.

10 S. Li, J. Li, M. Dong, S. Fan, T. Zhao, J. Wang and W. Fan, Chem. Soc. Rev., 2019, 48, 885.

11 X. Xie, J. Wang, J. Zheng, J. Huang, C. Ni, J. Cheng, Z. Hao and G. Ouyang, Anal. Chim. Acta, 2018, 1029, 30.

12 H. L. Nguyen, N. Hanikel, S. J. Lyle, C. Zhu, D. M. Proserpio and O. M. Yaghi, J. Am. Chem. Soc., 2020, 142, 2218.

13 J. Sun, A. Iakunkov, I. A. Baburin, B. Joseph, V. Palermo and A. V. Talyzin, Angew. Chem., Int. Ed., 2020, 59, 1087.

14 Y. Yuan and G. Zhu, ACS Cent. Sci., 2019, 5, 409.

15 T. Ben, H. Ren, S. Ma, D. Cao, J. Lan, X. Jing, W. Wang, J. Xu, F. Deng, J. M. Simmons, et al., Angew. Chem., Int. Ed., 2009, 48, 9457.

16 Y. Jin, Z. Li, L. Yang, J. Xu, L. Zhao, Z. Li and J. Niu, Anal. Chem., 2017, 89, 1290.

17 S. Grätz, S. Zink, H. Kraffczyk, M. Rose and L. Borchardt, Beilstein J. Org. Chem., 2019, 15, 1154.

18 S. Xu, Y. Luo and B. Tan, Macromol. Rapid Commun., 2013, 34, 471.

19 N. B. McKeown, P. M. Budd, K. J. Msayib, B. S. Ghanem, H. J. Kingston, C. E. Tattershall, S. Makhseed, K. J. Reynolds and D. Fritsch, Chemistry, 2005, 11, 2610.

20 A. I. Cooper, Adv. Mater., 2009, 21, 1291.

21 L. Pan, Q. Chen, J.-H. Zhu, J.-G. Yu, Y.-J. He and B.-H. Han, Polym. Chem., 2015, 6, 2478.

22 M.-Y. Wang, Q.-J. Zhang, Q.-Q. Shen, Q.-Y. Li and S.-J. Ren, Chin. J. Polym. Sci., 2020, 38, 151.

23 X.-J. Zhang, N. Bian, L.-J. Mao, Q. Chen, L. Fang, A.-D. Qi and B.-H. Han, Macromol. Chem. Phys., 2012, 213, 1575.

24 Z. Guo, P. Sun, X. Zhang, J. Lin, T. Shi, S. Liu, A. Sun and Z. Li, Chem.-Asian J., 2018, 13(16), 2046-2053.
25 O. S. Taskin, B. Kiskan, A. Aksu, N. Balkis and Y. Yagci, Des. Monomers Polym., 2015, 18, 567.

26 F. M. Wisser, K. Eckhardt, D. Wisser, W. Böhlmann, J. Grothe, E. Brunner and S. Kaskel, Macromolecules, 2014, 47, 4210.

27 E. Preis, N. Schindler, S. Adrian and U. Scherf, ACS Macro Lett., 2015, 4, 1268.

28 J. Wang, P. Zhang, C. Wang, X. Zheng, Y. Zhao, L. Li and S. Miao, Mater. Des., 2020, 186, 108371.

29 K. J. Msayib and N. B. McKeown, J. Mater. Chem. A, 2016, 4, 10110.

30 B. Li, Z. Guan, X. Yang, W. D. Wang, W. Wang, I. Hussain, K. Song, B. Tan and T. Li, J. Mater. Chem. A, 2014, 2, 11930.

31 M. Grzybowski, B. Sadowski, H. Butenschön and D. T. Gryko, Angew. Chem., Int. Ed., 2019, 59(8), 2998-3027.

32 D. Leistenschneider, K. Wegner, C. Eßbach, M. Sander, C. Schneidermann and L. Borchardt, Carbon, 2019, 147, 43. 33 J. Andersen and J. Mack, Green Chem., 2018, 20, 1435.

34 D. Tan and T. Friščic, Eur. J. Org. Chem., 2018, 18-33.

35 S. Ahmed and S. A. Chaudhry, Composites for Environmental Engineering, Wiley, 2019.

36 C. Bolm and J. G. Hernández, Angew. Chem., Int. Ed., 2019, $58,3285$.

37 J. L. Howard, Q. Cao and D. L. Browne, Chem. Sci., 2018, 9, 3080.

38 T. Friščić, C. Mottillo and H. M. Titi, Angew. Chem., Int. Ed., 2019, 59(3), 1018-1029.

39 S. L. James, C. J. Adams, C. Bolm, D. Braga, P. Collier, T. Friščić, F. Grepioni, K. D. M. Harris, G. Hyett, W. Jones, et al., Chem. Soc. Rev., 2012, 41, 413.

40 M. J. Muñoz-Batista, D. Rodriguez-Padron, A. R. PuenteSantiago and R. Luque, ACS Sustainable Chem. Eng., 2018, 6, 9530.

41 S. Grätz, M. Oltermann, E. Troschke, S. Paasch, S. Krause, E. Brunner and L. Borchardt, J. Mater. Chem. A, 2018, 6, 21901.

42 S. Grätz, D. Beyer, V. Tkachova, S. Hellmann, R. Berger, X. Feng and L. Borchardt, Chem. Commun., 2018, 54, 5307.

43 P. Zhang, X. Jiang, S. Wan and S. Dai, J. Mater. Chem. A, 2015, 3, 6739.

44 B. P. Biswal, S. Chandra, S. Kandambeth, B. Lukose, T. Heine and R. Banerjee, J. Am. Chem. Soc., 2013, 135, 5328.

45 E. Troschke, S. Grätz, T. Lübken and L. Borchardt, Angew. Chem., Int. Ed., 2017, 56, 6859.

46 P. Zhang and S. Dai, J. Mater. Chem. A, 2017, 5, 16118.

47 S. G. Patnaik, R. K. Gagan, R. Vedarajan, A. Yamaguchi, M. Miyauchi and N. Matsumi, J. Electrochem. Soc., 2018, 165, J3166-J3172.

48 P. O. Miranda, R. M. Carballo, M. A. Ramírez, V. S. Martín and J. I. Padrón, ARKIVOC, 2006, 2007, 331.

49 R. Tomifuji, K. Maeda, T. Takahashi, T. Kurahashi and S. Matsubara, Org. Lett., 2018, 20, 7474.

50 T. Saito, Y. Nishimoto, M. Yasuda and A. Baba, J. Org. Chem., 2006, 71, 8516. 